\title{
Numerical model of electrical potential within a human head
}

\author{
P.E. Rasser* M.D. Teubner* C.R. Clark $^{\dagger}$ \\ (Received 7 August 2000)
}

\begin{abstract}
Areas of activity within the human brain can result from a variety of external stimuli. This paper discusses a technique for determining the location of sources of this activity as a result of some stimulus.
\end{abstract}

*Department of Applied Mathematics and Centre for Applied Modelling in Water Engineering, University of Adelaide, Australia.

mailto:prasser@maths.adelaide.edu.au and mailto:mteubner@maths.adelaide.edu.au

${ }^{\dagger}$ Cognitive Neuroscience Laboratory and School of Psychology, Flinders University, AUstraliA. mailto:richard.clark@flinders.edu.au

${ }^{0}$ See http: //anziamj . austms .org.au/V42/CTAC99/Rass for this article and ancillary services, (C) Austral. Mathematical Soc. 2000. Published 27 Nov 2000. 
Source location inside a human head is determined numerically by way of coupled forward and inverse models. The forward model determines the electrical potential on the scalp as a result of a source inside the brain, and the inverse model uses measured values of electrical potential on the scalp to determine the location of the source.

Previous models have used structural magnetic resonance imaging (SMRI) for determining the boundaries of the larger structures of the head, these being the white and grey matter of the brain, cerebrospinal fluid, skull and scalp. They have also assumed a homogeneous conductivity for each of these units. It has been shown, however, that the electrical conductivities of these substructures can vary considerably.

A three-dimensional, finite-difference model of the human head is developed and compared against a known analytical solution. SMRI data is then used to define the grid size of the finite-difference model and the conductivities within these grid points. The forward model presented here uses conductivities obtained by assuming a functional relationship between the intensity values of the sMRI and conductivity.

\section{Contents}

2.1 Boundary conditions . . . . . . . . . . . . . . . . C1223

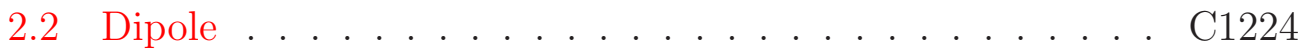


2.3 The analytical solution to the steady-state model . . . . . C1224

3 Numerical Model

C1226

4 A Three-Sphere Steady-State Model

C1227

4.1 Numerical model . . . . . . . . . . . . . . . . . C1228

4.2 Verification of numerical model . . . . . . . . . . . C1228

$5 \quad$ Numerical model using sMRI data

C1232

6 Conclusions and Recommendations

C1235

References

C1235

\section{Introduction}

Neuronal activity inside the human brain gives rise to electrical potential which flows through the various regions of the head to the scalp. Recorded electrical potential on the scalp is obtained using data from an electroencephalogram (EEG).

By assuming the head is a volume conductor of piecewise continuous regions of constant conductivity, three-sphere models have been developed [9, 10], which partition the head into the brain, skull and scalp. Four-sphere 
models have also been developed [2], which also take into account the cerebrospinal fluid (CSF). In these analytical models, each of the concentric spheres is given a uniform conductivity value.

More recently with the wider use of structural magnetic resonance imaging (sMRI), models of the flow of electrical potential have used real head geometry, as opposed to assuming concentric spheres. Models that use sMRI for the structure of the head have most commonly used finite-element techniques $[7,12]$ or boundary element methods $[5,12]$. In these models, segmentation routines have been used to define the boundaries of the different tissue types from the sMRI.

Data from sMRI is represented in voxels, with each voxel assigned an intensity value. For low resolution sMRI, 17 images are produced representing adjacent slices throughout the brain, with each image being developed from a grid of $128 \times 128$ intensity values. This gives each voxel an approximate dimension of $4 \mathrm{~mm} \times 4 \mathrm{~mm} \times$ by $7 \mathrm{~mm}$. For high resolution sMRI, 164 images are produced with each image having a resolution of $256 \times 256$ values. Voxels from this high resolution sMRI have dimensions of approximately $1 \mathrm{~mm} \times$ $1 \mathrm{~mm} \times 1 \mathrm{~mm}$.

Segmentation of the various units of the human head is performed by normalising the sMRI data and setting an intensity range as well as a starting point inside the segmenting region. All voxels outside this intensity range are excluded and a boundary of inclusion assigned [3,6]. One problem associated with segmentation routines lies with partial voluming of the voxels. At 
boundary regions like the interface between the grey matter of the cerebral cortex and the CSF, an intensity value would be assigned that reflects the intensity of both of these materials. Given that sMRI data is uniformly divided into a grid, by assuming a functional relationship between the intensity from the sMRI and conductivity, a uniform grid of conductivity values may be obtained.

The forward model discussed in the paper forms part of a larger assessment of the sources of cognitive function within the brain. It is intended that the forward model be coupled with an inverse model $[11,13]$ for the determination of source location based on real EEG and SMRI data. This problem, however, is ill-posed as many solutions of source location may generate a satisfactory match to the recorded EEG. This being the case, an additional imaging technique, functional magnetic resonance imaging (fMRI), which contributes information about the loci of functional activity in the brain, is intended to constrain the solution domain of the inverse model.

Presented here is a three-dimensional, alternating direction implicit finitedifference method to calculate the flow of electrical potential through the head. This model can use a grid resolution as defined by the sMRI and it is intended that conductivity be determined as a functional relationship of the intensity obtained from sMRI. This three-dimensional model is verified against a known analytical solution. 


\section{The Governing Equation}

The three-dimensional equation governing the flow of electrical potential in a non-homogeneous medium [8] is given by

$$
\frac{\partial}{\partial x}\left(K_{x} \frac{\partial \phi}{\partial x}\right)+\frac{\partial}{\partial y}\left(K_{y} \frac{\partial \phi}{\partial y}\right)+\frac{\partial}{\partial z}\left(K_{z} \frac{\partial \phi}{\partial z}\right)=\frac{\partial \phi}{\partial t}+Q
$$

where $x, y, z$ are Cartesian coordinates $(\mathrm{mm}), t$ is time (seconds), $\phi$ is the electrical potential (millivolts), $K_{x}, K_{y}, K_{z}$ are electrical conductivities in the $x, y, z$ directions respectively $\left(\mathrm{mm}^{2} / \mathrm{sec}\right), Q$ is the net source/sink of potential (millivolts/sec).

\subsection{Boundary conditions}

It is assumed that there is no flow of potential out from the modelled region, so that

$$
\frac{\partial \phi}{\partial n}=0
$$

on the outer surface, where $n$ is the direction normal to the outer surface. 


\subsection{Dipole}

A dipole is taken to be the unit of source activity and assumed to consist of both a source and a sink [8]. The source adds electrical potential to the system at a point location and the sink removes electrical potential from the system [8]. A dipole has strength, orientation and location.

\subsection{The analytical solution to the steady-state model}

Rush and Driscoll [10] determined a steady-state analytic solution for Equation (1) for the case of concentric spheres with varying electrical conductivities. Their solution is given by

$$
\phi(r, \theta)=\frac{A_{1}}{6 \pi \sigma_{t} c^{2}}\left[2 \frac{r}{c}+\left(\frac{c}{r}\right)^{2}\right] I D \cos \theta,
$$

where $r$ is the distance from the centre of the dipole to the measurement of potential, $\theta$ is the angle made with the line joining the centre of the dipole and measurement location and the $y$-axis, as shown in Figure $1, I$ is the strength of the source, $D$ is the distance between the source and the sink, and

$$
\begin{aligned}
& A_{1}=\left(\frac{27}{2}\right)\left[\left(\frac{\sigma_{b}}{\sigma_{s}}+2\right)\left(\frac{\sigma_{s}}{\sigma_{t}}+2\right)+2\left(\frac{\sigma_{b}}{\sigma_{s}}-1\right)\left(\frac{\sigma_{s}}{\sigma_{t}}-1\right)\left(\frac{a}{b}\right)^{3}\right. \\
& \left.+2\left(\frac{\sigma_{s}}{\sigma_{t}}-1\right)\left(\frac{\sigma_{b}}{\sigma_{s}}+2\right)\left(\frac{b}{c}\right)^{3}+\left(\frac{\sigma_{b}}{\sigma_{s}}-1\right)\left(\frac{4 \sigma_{s}}{\sigma_{t}}+2\right)\left(\frac{a}{c}\right)^{3}\right]^{-1},
\end{aligned}
$$



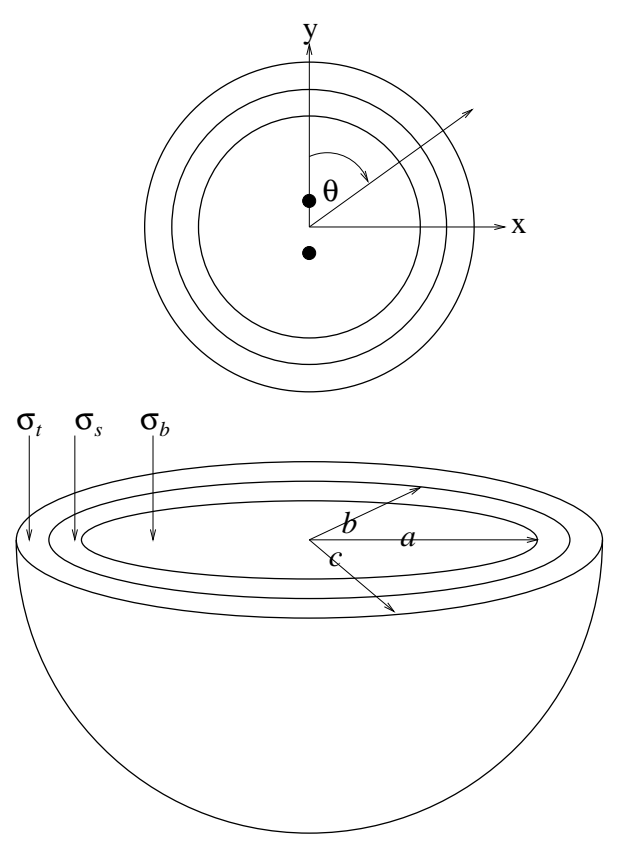

FiguRE 1: Diagram showing the three concentric spheres and some of the parameters of the analytic model. The source and sink are shown above with their midpoint being the origin. 
where $\sigma_{b}, \sigma_{s}$ and $\sigma_{t}$ are the conductivities of the inner, middle and outer spheres respectively, with radii $a, b$ and $c$. Figure 1 shows some of the parameters of the concentric sphere model.

\section{$3 \quad$ Numerical Model}

To solve Equation (1) numerically the Douglas-Rachford Alternating Direction Implicit (ADI) finite-difference method was used [4]. The finite-difference technique is applied over the solution domain by using a rectangular grid with spacings of $\Delta x, \Delta y, \Delta z$ and $\Delta t$ in the $x, y, z$ and $t$ directions respectively, where $\Delta x, \Delta y$ and $\Delta z$ are measured in $\mathrm{mm}$ and $\Delta t$ is measured in seconds. Using the notation $x_{i}=i \Delta x, y_{j}=j \Delta y, z_{k}=k \Delta z$ and $t_{n}=n \Delta t$ for integer values of $i, j, k$ and $n$, the electrical potential at the $(i, j, k)^{t h}$ grid point at time $t_{n}$ can be written as $\phi_{i, j, k}^{n}=\phi\left(x_{i}, y_{j}, z_{k}, t_{n}\right)$.

Using the Douglas-Rachford ADI finite-difference method, Equation (1) is split over three time levels to give

$$
\begin{gathered}
\frac{\partial}{\partial x}\left(K_{x} \frac{\partial \phi}{\partial x}\right)_{i, j, k}^{n+1 / 3}+\frac{\partial}{\partial y}\left(K_{y} \frac{\partial \phi}{\partial y}\right)_{i, j, k}^{n}+\frac{\partial}{\partial z}\left(K_{z} \frac{\partial \phi}{\partial z}\right)_{i, j, k}^{n}=\left(\frac{\partial \phi}{\partial t}\right)_{i, j, k}^{n}+Q_{i, j, k}^{n} \\
\frac{\partial}{\partial y}\left(K_{y} \frac{\partial \phi}{\partial y}\right)_{i, j, k}^{n+2 / 3}=\frac{\partial}{\partial y}\left(K_{y} \frac{\partial \phi}{\partial y}\right)_{i, j, k}^{n}+\left(\frac{\partial \phi}{\partial t}\right)_{i, j, k}^{n+1 / 3}
\end{gathered}
$$




$$
\frac{\partial}{\partial z}\left(K_{z} \frac{\partial \phi}{\partial z}\right)_{i, j, k}^{n+1}=\frac{\partial}{\partial z}\left(K_{z} \frac{\partial \phi}{\partial z}\right)_{i, j, k}^{n}+\left(\frac{\partial \phi}{\partial t}\right)_{i, j, k}^{n+2 / 3},
$$

where the spatial derivatives of Equations (5)-(7) are evaluated using a blockcentred finite-difference formula and the time derivatives approximated using a forward-time finite-difference formula incorporating a time step in each case of $\Delta t / 3$. The Thomas algorithm was used to invert the matrix developed from each of Equations (5)-(7) as they contain the unknowns $\phi_{i-1, j, k}^{n+1 / 3}$, $\phi_{i, j, k}^{n+1 / 3}, \phi_{i+1, j, k}^{n+1 / 3} ; \phi_{i, j-1, k}^{n+2 / 3}, \phi_{i, j, k}^{n+2 / 3}, \phi_{i, j+1, k}^{n+2 / 3} ;$ and $\phi_{i, j, k-1}^{n+1}, \phi_{i, j, k}^{n+1}, \phi_{i, j, k+1}^{n+1}$ respectively.

\section{A Three-Sphere Steady-State Model}

The model of three concentric spheres presented in Section 2.3 has been developed with $a=80 \mathrm{~mm}, b=90 \mathrm{~mm}$ and $c=100 \mathrm{~mm}$. The electrical conductivities of the inner, middle and outer spheres have been chosen to be $\sigma_{b}=100, \sigma_{s}=1.25$, and $\sigma_{t}=100$ respectively. The conductivities are in the ratio of $1: 1 / 80: 1$; as used by [1]. A source and a sink of potential 12000 and -12000 millivolts respectively are placed $10 \mathrm{~mm}$ apart in the centre of the concentric spheres oriented parallel to the $y$-axis and lying on the $x y$-plane. 


\section{$4.1 \quad$ Numerical model}

To obtain a solution for the three-sphere steady-state model, a grid of dimensions $53 \times 53 \times 53$ was used. Each grid spacing in the $x, y$, z-direction (i.e. $\Delta x, \Delta y$ and $\Delta z$ ) corresponded to $5 \mathrm{~mm}$ in length. A dipole was modelled using a source located at the grid point $(27,28,27)$ and a sink located at $(27,26,27)$ (effectively producing a dipole of length $10 \mathrm{~mm}$ and orientation of $90^{\circ}$ with the $x$-axis), with strength of 12000 and -12000 millivolts respectively. The model was run four times until steady-state, which was reached when the largest difference in potential between any grid point over a time step was smaller than a tolerance criteria of $10^{-10}, 10^{-8}, 10^{-6}$ and $10^{-4}$ millivolts respectively.

\subsection{Verification of numerical model}

Figure 2 shows the comparison between the results obtained from the numerical model and from the analytical solution in the $y$-direction, with the dipole located at the centre of the grid. The results from using the four different tolerance criteria are shown. Because of the discretisation, the first value from the numerical model is located at $5 \mathrm{~mm}$ and then at $5 \mathrm{~mm}$ intervals along the $y$-axis. Figure 2 shows excellent agreement between the analytical and numerical solutions for all tolerance values. Figure 3 uses the same data as Figure 2, with the scale on the $y$-axis changed. Figure 3 shows very good 


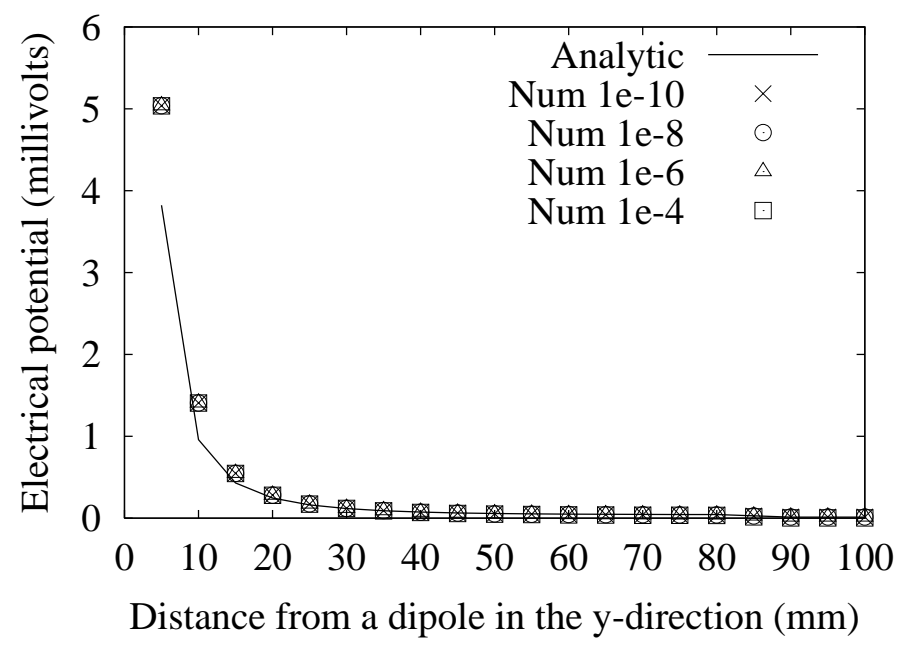

Figure 2: Comparison of analytical and numerical solutions in the $y$ direction for the three-sphere steady-state model. Num $1 \mathrm{e}^{-10}$, etc., refers to the numerical solution with a tolerance criteria of $1 \times 10^{-10}$, etc. 


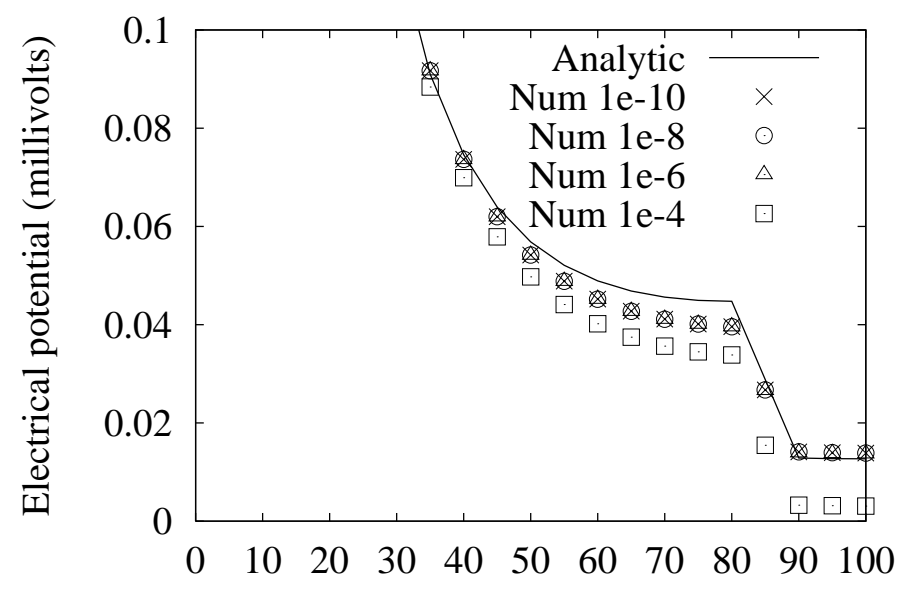

Distance from a dipole in the $y$-direction $(\mathrm{mm})$

Figure 3: Comparison of analytical and numerical solutions in the $y$ direction for the three-sphere steady-state model. Num $1 \mathrm{e}^{-10}$, etc., refers to the numerical solution with a tolerance criteria of $1 \times 10^{-10}$, etc. 


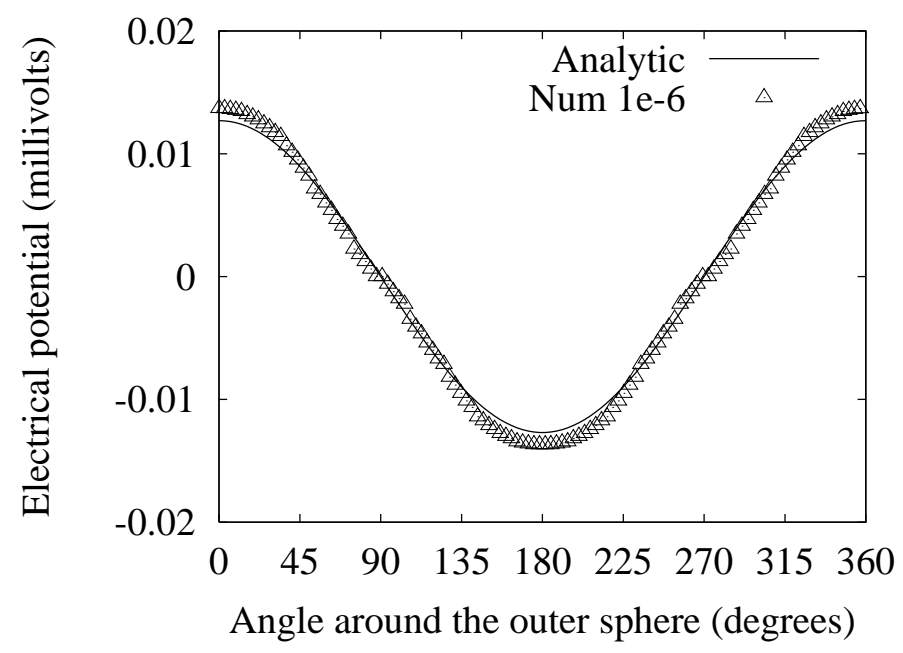

FiguRE 4: Comparison of analytical and numerical solutions on the outer sphere for the three-sphere steady-state model. 
agreement between the analytical solution and the numerical solution using tolerance criteria smaller than $10^{-4}$ millivolts. This suggested that a tolerance of $10^{-6}$ would be sufficient in all further simulations. Figure 3 indicates the variability of electrical potential $\phi$ when moving radially out from the dipole. Values of $\phi$ decrease exponentially within the inner sphere; across the middle sphere where the conductivity is reduced by a factor of 80 , the variation is effectively linear, and the variation is almost constant throughout the outer sphere.

As the forward model is to be used to produce results that will be compared with EEG data, the numerical solutions from the spherical model of greatest interest are located on the outside of the largest sphere. Figure 4 shows a comparison of the analytical and numerical solutions for the points which define the outer surface on the $z=27$ plane. Once again very good comparisons between the two solutions are achieved.

\section{$5 \quad$ Numerical model using sMRI data}

Intensity values from a low resolution SMRI scan were transformed to conductivities by assuming the functional relationship

$$
K_{x}(x, y, z), K_{y}(x, y, z), K_{z}(x, y, z)=(1493-\operatorname{Intensity}(\mathrm{x}, \mathrm{y}, \mathrm{z}))^{2} / 500,
$$

for all intensity values greater than zero. This relationship was assumed after a filtering was applied to the intensities to remove spurious data outside the 

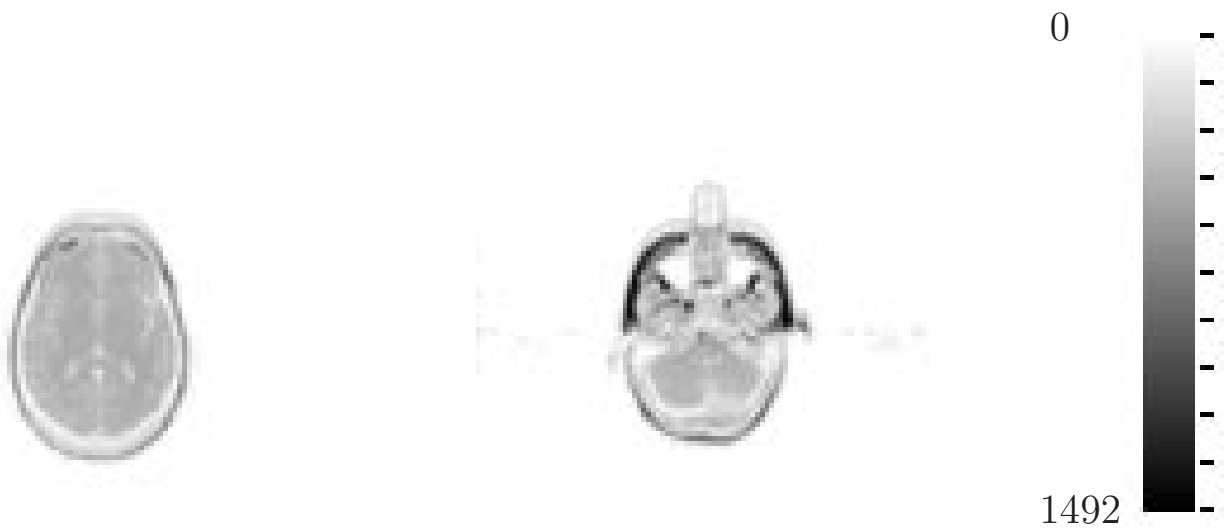

FiguRE 5: The $8^{\text {th }}$ and $17^{\text {th }}$ slices from a low resolution sMRI scan.

head region. The intensities from the sMRI scan ranged from 0 to 1492. The relationship presented in Equation (8) is based on the assumption that the skull (having the darkest intensity and the highest intensity value) has the lowest conductivity value, and all other intensity values follow a similar relationship. Figure 5 shows a grey scale representation of the $8^{\text {th }}$ and $17^{\text {th }}$ slices of the low resolution sMRI scan.

Although the low resolution sMRI data is in a $128 \times 128 \times 17$ grid format, the intensity values containing the physical head lie within a $69 \times 69 \times 17$ grid. A grid of these dimensions was used for the forward model in order to reduce the computational effort. A source located at $(34,35,8)$ and a sink at 

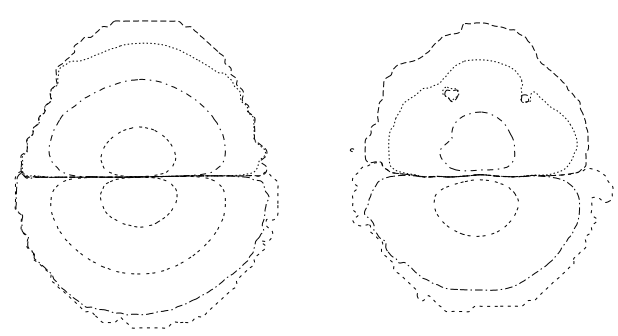

FiguRE 6: Equipotential contours of the $8^{\text {th }}$ and $17^{\text {th }}$ slices from the numerical model.

$(34,33,8)$ with strengths of 12,000 and $-12,000$ millivolts respectively were used to represent a dipole. The forward model was run to steady-state with a tolerance of $10^{-6}$ millivolts. Figure 6 shows equipotential contours for the $8^{\text {th }}$ and $17^{\text {th }}$ slices related to the sMRI data presented in Figure 5. These slices show the distribution of electrical potential generated by the dipole. The outer contours indicate the potential that can be compared with measured EEG. 


\section{Conclusions and Recommendations}

A three-dimensional ADI finite-difference model of the movement of electrical potential within the human head has been developed and verified against a three concentric sphere analytical solution. This finite-difference model has also been applied to a real head geometry obtained from low resolution sMRI, where it was assumed that the conductivity of the head is a function of the tissue intensity. The results from this model can be used to compare real EEG data for the problem of source localisation. Further work still needs to be done with the relationship between the intensity and conductivity, as well as moving to high resolution sMRI to more accurately define the model region and conductivities. It it also intended that sensitivity on the stability of the finite-difference technique be analysed, and that an inverse model be incorporated for source localisation.

Acknowledgements: The authors acknowledge the contribution of Andrew Lewis in preparing the sMRI scans; Karl Sammut, Fang Po He, Bill Moran and Murk Bottema for their intellectual contributions; and a grant for financial support from the Australian Research Council.

\section{References}


[1] B.N. Cuffin. A method for localizing EEG sources in realistic head models. IEEE Trans. Biomed. Eng., 42(1):68-71, 1995. C1227

[2] B.N. Cuffin and D. Cohen. Comparison of the magnetoencephalogram and electroencephalogram. Electroencephalography and Clinical Neurophysiology, 47:132-146, 1979. C1221

[3] A.M. Dale, B. Fischl, and M.I. Sereno. Cortical surface-based analysis I: Segmentation and surface reconstruction. NeuroImage, 9:179-194, 1999. C1221

[4] J. Douglas Jr. and H.H. Rachford Jr. On the numerical solution of heat conduction problems in two and three space variables. Trans. Am. Math. Soc., 82:421-439, 1956. C1226

[5] D.J. Fletcher, A. Amir, D.L. Jewett, and G. Fein. Improved method for computation of potentials in a realistic head shape model. IEEE Trans. Biomed. Eng., 42(11):1094-1103, 1995. C1221

[6] M. Joshi, J. Cui, K. Doolittle, S. Joshi, D. Van Essen, L. Wang, and M.I. Miller. Brain segmentation and the generation of cortical surfaces. NeuroImage, 9:461-476, 1999. C1221

[7] G. Marin, C. Guerin, S. Baillet, L. Garnero, and G. Meunier. Influence of skill anisotropy for the forward and inverse problem in EEG: Simulation studies using FEM on realistic head models. Human Brain Mapping, 6:250-269, 1998. C1221 
[8] P. Nunez. Electric Fields of the Brain. Oxford University Press, 1981. C1223, C1224, C1224

[9] R.D. Pascual-Marqui, C.M. Michel, and D. Lehmann. Low resolution electromagnetic tomography: a new method for localizing activity in the brain. Int. J. of Psychophysiology, 18:49-65, 1994. C1220

[10] S. Rush and D.A. Driscoll. EEG electrode sensitivity - an application of reciprocity. IEEE Trans. Biomed. Eng., BME-16(1):15-22, 1969. C1220, C1224

[11] P.E. Rasser and M.D. Teubner. Groundwater model of the Willunga basin using automated calibration. In B.J. Noye, M.D. Teubner, and A.W. Gill, editors, Computational Techniques and Applications: CTAC97, pages 569-576, Singapore, 1998. World Scientific. C1222

[12] G.V. Simpson, M.E. Pflieger, J.J. Foxe, S.P. Ahlfors, J. Vaughan Jr., H.G. Hrabe, R.J. Ilmoniemi, and G. Lantos. Dynamic neuroimaging of brain function. Journal of Clinical Neurophysiology, 12(5):432-449, 1995. C1221, C1221

[13] M.D. Teubner, P.E. Rasser, and B.J. Noye. Systematic approach to calibrating hydrodynamic numerical models. In R.L. May and A.K. Easton, editors, Computational Techniques and Applications: CTAC95, pages 763-770, 1996. C1222 